\title{
Moxon Antenna Array Design and Pattern Synthesis Technique
}

\author{
*1Suad Başbuğ \\ ${ }^{1}$ Department of Electrical and Electronics Engineering, Nevsehir Haci Bektas Veli University, Nevsehir, Turkey, \\ suad@nevsehir.edu.tr@iD \\ Research Paper \\ Arrival Date: 19.11.2018 \\ Accepted Date: 29.04.2019
}

\begin{abstract}
A four-element moxon antenna array is proposed in this paper. Its directive properties are shown with simulations and compared with the classical dipole array. In order to synthesize the pattern of moxon array, a hybrid method that consists of differential evolution (DE) and numerical electromagnetics code version 2 (NEC2) is also introduced. Basically, the cost function used by DE is designed with the help of NEC2 simulations to achieve more realistic optimum antenna array designs. Patterns having one and double sidelobe suppression at predetermined angles are successfully synthesized by using this method whereas the gain value is maintained properly. Robustness of the proposed method is also examined. For this aim, multiple executions of the method implementation are performed under same conditions. The results show that the method introduced in this paper is very stable in terms of the radiation pattern and convergence curves.
\end{abstract}

Keywords: Directive antenna arrays, metaheuristic algorithms, moxon antenna, NEC2, pattern synthesis.

\section{INTRODUCTION}

One of the main advantages of the antenna arrays is that they can produce directive radiation patterns. Antenna array elements can be any type of antennas. Simple array elements have two basic advantages which are simple construction and easy calculation. Usage of dipoles as array elements is a good idea since the dipole is one of the simplest types of antennas [1]. However, linear dipole antenna arrays radiate in both perpendicular directions to the array axis equally and symmetrically when its array elements are uniformly excited. It is a clear disadvantage when it is necessary to radiate only one direction. A reflector element might be a good solution for each element as used in Yagi-Uda antennas to focus the main beam of the radiation to one determined direction. In this context, Moxon antennas are in the group of two elements Yagi-Uda antennas.

Moxon antennas have been chosen for several types of applications owing to their advantages [2-4]. First of all, they are compact compared to their alternatives [2]. As a second advantage, they can produce patterns which have remarkable front-to-back ratio [3]. Lastly, one does not need to use an additional impedance matching circuit in general when using a Moxon antenna [4].

In this paper, we propose a moxon antenna array design to produce a directive radiation pattern. The design also includes single and double suppression facilities on the pattern. The sidelobe suppression is done by controlling amplitude and phase parameters of array elements. Any metaheuristic algorithm can be used to calculate the amplitude and phase values. In this work, differential evolutionary (DE) [5] algorithm is chosen for this purpose.A comprehensive technique is used to optimize antenna parameters. Classical techniques [6-8] generally use the array factor formula to shape the patterns. This obviously provides easy calculation advantages. However, the optimization methods that use the cost function constructed by considering only the array factor fall short to take account of some important parameters such as mutual coupling and impedance bandwidth. In the literature, in order to optimize a twin polygonal loop antenna array [9] and a dual monopole antenna having a spiral geometry [10] with high gain and minimum reflection, the original source code of Numerical Electromagnetics Code Version 2 (NEC2) [11] is modified in $\mathrm{C}++$ to let it work with particle swarm optimization algorithm. In our work, NEC2++ [12] is utilized as a library without modifying the original source code. There are two main contributions of the work in this paper.

First, contrary to the other classical techniques that use the It means that the objective function is based on the NEC2 simulation results in place of simple array factor formula outcomes. Second, in this work, any modification is not performed on NEC original codes. Instead, the optimization parameters are passed to the NEC2 engine as a black box function to obtain the simulation results. 
In this way, the proposed method is able to keep the overall computational method from the programming language dependency. A custom software implementation developed in Python gains access to the antenna simulation library by importing NEC2++ module composed originally in $\mathrm{C}++$. In this implementation, DE sends the main geometric and electrical parameters of the moxon antenna to the NEC2++ simulator. An array factor formula is not needed since NEC2++, as a variant of NEC2, uses the method of moments technique for its calculations. The cost function taken into account by DE is developed by considering the simulation results of NEC2++. This cycle is performed in every iteration in the optimization process.

\section{MOXON ANTENNA ARRAY}

A moxon antenna comprises two parts: A driven element fed at the center and a reflector element which is longer than the driven element as shown in Fig. 1. Driven element is in fact a bent dipole antenna. The moxon antenna array proposed in this paper is illustrated in Fig. 2. The moxon antennas as array elements are arranged in a straight line. The reflector parts of the array elements are aligned parallel to $z$-axis. The distances between the elements are equal. The direction of main beam is the positive $y$-axis. The moxon array sample with four elements in Fig. 2 is designed for the examples of this paper. The numerical details are given in the simulation results section.

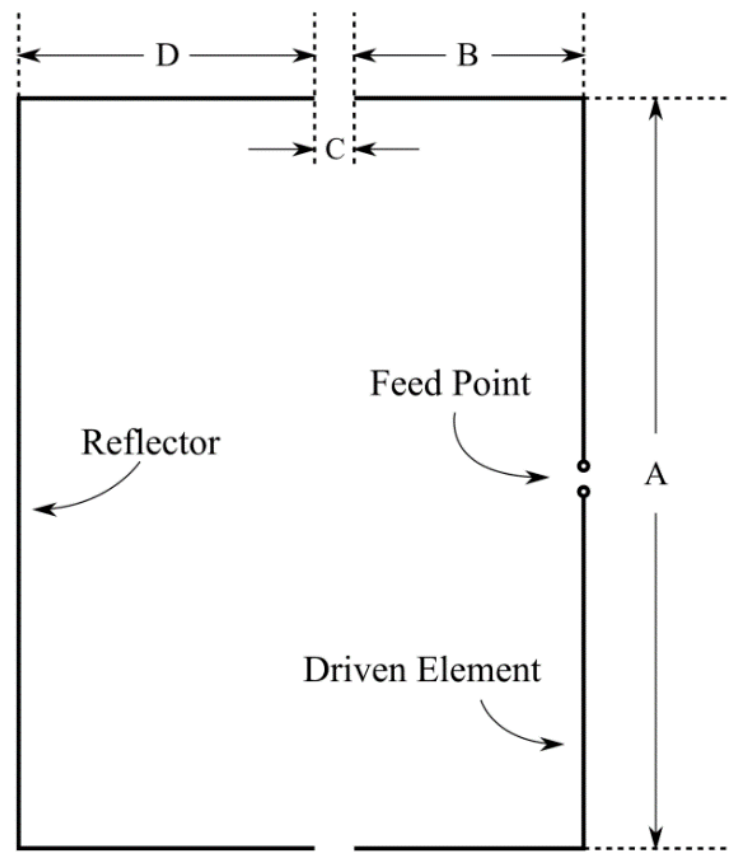

Figure 1. Moxon antenna

A scheme is given in Fig. 3 to explain the excitation method of the proposed moxon array. The examples of the single and double sidelobe suppression on the pattern are performed by controlling the amplitude and phase values of the array elements.
In the scheme, the radio frequency signal is divided into four channels by a splitter. These four signals have same amplitude and phase values before the control unit. The control unit have attenuators and phase shifters for each channel. In order to shape array patterns electronically, the amplitude and phase values of the signals that goes to the antennas are changed by the attenuators and phase shifters. For the sake of simplicity, the other details such as microcontroller unit and amplifiers are not shown in Fig. 3.

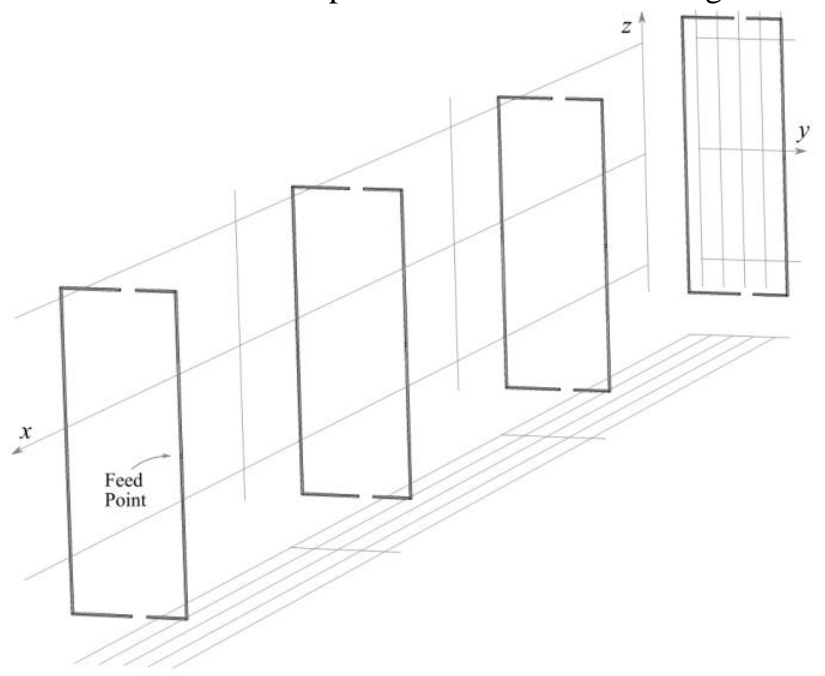

Figure 2. Moxon antenna array

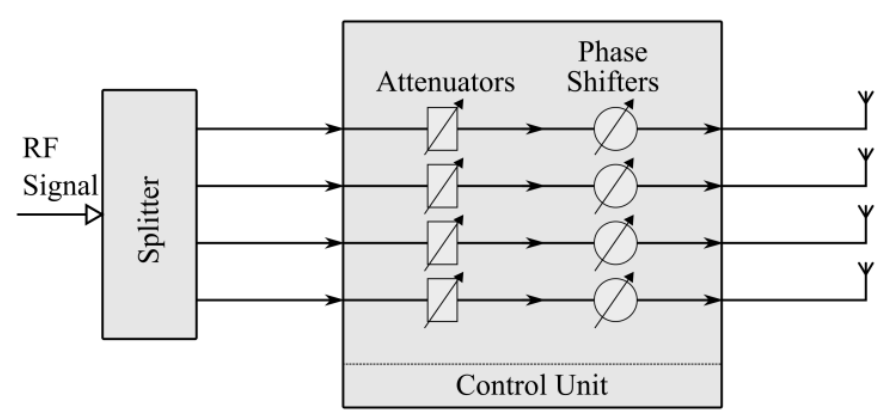

Figure 3. Control unit for signal attenuation and phase shifting

\section{PATTERN SYNTHESIS METHOD}

The method proposed in this paper for pattern synthesis of moxon antenna array exploits the benefits of NEC2 antenna simulation engine and DE optimization algorithm jointly. In this method, DE optimization algorithm uses a cost function to shape the desired array pattern. The cost function is composed of the restrictions on the pattern obtained from the results of NEC2++ which is a software library version of original NEC2. The cost function can be represented mathematically as follows:

$C=\left(G_{d}-G_{0}\right)^{2}+\sum_{k=1}^{K}\left(S_{k}-S_{d}\right)^{2}$ 
where $G_{d}$ and $G_{0}$ are the desired and calculated gain of the antenna array, respectively. In a similar way, $S_{d}$ and $S_{k}$ are the desired and calculated sidelobe levels at the predetermined angles, respectively. $G_{d}$ must be greater than $G_{0}$ in this calculation, otherwise the relevant part of the calculation is zero. Similarly, $S_{k}$ must be greater than $S_{d}$, since the calculated sidelobe cannot be acceptable higher than the limits. The main target of DE algorithm is to find optimum parameters which make the value of the cost function minimum. The gain and sidelobe calculations are performed by means of NEC $2++$ module.

NEC2 is the second version of Numerical Electromagnetic Code (NEC) software released under public domain. It provides useful tools to solve electromagnetic problems, which are especially related to antennas [11]. The software is based on the numerical solutions of electric-field and magnetic field integral equations obtained by method of moments. NEC mainly utilizes the electric-field integral equations to model electromagnetic response of thin-wire structures.

The magnetic-field integral equations are specialized for the large smooth surfaces in NEC. The models of NEC can also handle with perfect and imperfect conductors, lumped element loading, and ground planes. After the solution, it is possible to achieve current and charge densities, near-field and far-field results, impedance and admittance, gain, directivity and power budget calculated by NEC. NEC2 has same core features with NEC. Although NEC2 also owns some different properties from that of NEC, the features of NEC2 used in this work is not different from NEC. In this paper, we use NEC2++ [12] to simulate the moxon antenna array programmatically in order to construct the cost function and guide DE algorithm.

NEC2++ mirrors all the capabilities of the original NEC2. $\mathrm{NEC} 2++$ is coded with $\mathrm{C}++$ and released under the general public license. NEC2++ also offers an opportunity for utilizing NEC2 engine directly within computer programs developed by using Python, Ruby, $\mathrm{C}$ and $\mathrm{C}++$ Languages. This provides very good flexibility for custom applications. In this paper, we employ Python programming language to access NEC2++ library.

DE is a population based metaheuristic algorithm [5]. In general, the population members search an optimal solution in the solution space by exploiting the position information of the other members. This information exchange among the members is performed by a simple mathematical formula. Each member has a chance as a trial member to benefit from this mutation phase at every single iteration.

The mutant member built by the means of the information sharing substitutes for the trial member if it has a better performance than the trial member in terms of cost function. Randomization is utilized at three stages during the optimization process: scattering population member at the beginning, selecting random members to apply the mutation formula and performing recombination. Termination criteria may be a maximum iteration number or an optimal solution that fulfills the requirements. DE algorithm can be formally described as follows:

$N P$ : Number of population members

$F$ : Scaling factor in $(0,2]$

$C R$ : Crossover rate in $(0,1)$

$\operatorname{random}()$ is a function that returns a real number in $[0$, 1)

Let $p \in \mathbb{R}^{n}$ be trial member

$K: \mathbb{R}^{n} \rightarrow \mathbb{R}$, the cost function

- Initialization

$\checkmark$ Distribute all population members randomly throughout the solution space.

$\checkmark$ Calculate the cost function values of all members.

- For each iteration until the termination criteria is satisfied.

- For each member as a trial member $x$

- Select three random members $(a, b$ and $c$ ) different from each other and the trial member.

- For each $i$ dimension to build a mutant member $m$ : $m_{i}=a_{i}+F\left(b_{i}-c_{i}\right)$ if $\operatorname{random}()<$ $C R$, otherwise $m_{i}=x_{i}$

- Calculate the cost function value of the mutant member

- If the cost function value of mutant member $m$ is better than that of trial member $x$, the trial member is replaced with the mutant member $m$.

Display the population member as an optimal solution, which has the best cost function value at the end of the optimization.

The positions of the population members of DE in our work corresponds amplitude and phase values of array elements. Our examples are built on the idea of four-element moxon array. Each array element has its own amplitude and phase values. Therefore, in this work, DE algorithm deals with a problem having eight real dimensions.

\section{SIMULATION RESULTS}

In order to show the performance of the moxon array and the proposed pattern synthesis method, we take account of a four-element antenna array as illustrated in Fig. 2. The center frequency is chosen as $433 \mathrm{MHz}$. The geometrical 
configuration is arranged by considering this frequency value. As an array element, the moxon antenna dimension values are calculated by means of MoxGen software [13].

The wire radius is set to $1.02616 \mathrm{~mm}$ (12 AWG). With these frequency and wire size inputs, MoxGen produces the following values for the geometrical parameters in Fig. 1: A $=248.3 \mathrm{~mm}, \mathrm{~B}=31.9 \mathrm{~mm} \mathrm{C}=12.6 \mathrm{~mm}$, and $\mathrm{D}=48 \mathrm{~mm}$. The moxon array used in this paper comprises four elements separated from each other with a $485.611 \mathrm{~mm}$ distance. This distance is computed by using the classical optimization tool of 4 nec2 [14].

Proposed array synthesis method is written in Python 2 language by using Spyder development environment. NEC2++ is integrated into the implementation as a library. $\mathrm{DE}$ algorithm has been used to alculate both amplitude and phase values of moxon array elements in order to place desired nulls on the pattern. 4nec2 is employed as the antenna simulator to analyze and illustrate the results calculated by the proposed method. First of all, traditional half-wave dipole (346 mm length) array and the proposed moxon array are compared to point out the higher gain and better front-to-back ratio of moxon array. Fig. 4 shows both the patterns of dipole and moxon antenna arrays.

The dipole array elements have the same wire radius and element spacing with the moxon array. It is clear from Fig. 4 that Moxon Array has higher gain than dipole antenna. The gain of moxon array is $13.1 \mathrm{dBi}$ whereas that of dipole array is $10.5 \mathrm{dBi}$, the front-to-back ratio of moxon array is $11.7 \mathrm{~dB}$. Both patterns in 3D can be viewed in Fig. 5. From Fig.5, it can be inferred that the azimuth $(\theta)$ direction radiation values are in harmony with 2D plot of radiation pattern in Fig. 4.

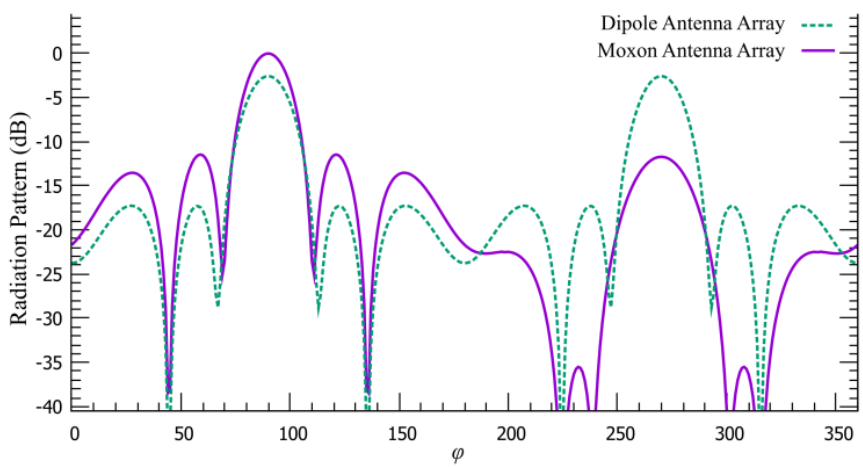

Figure 4. Pattern comparison of uniformly exited dipole and moxon antenna array.

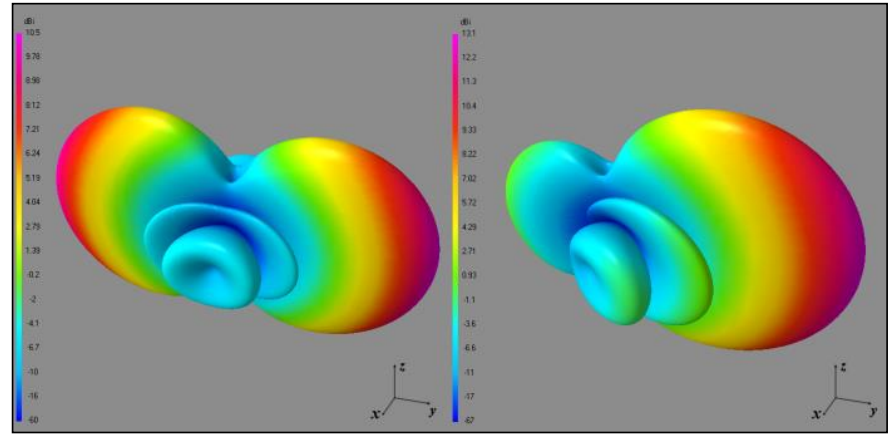

(a)

(b)

Figure 5. Patterns of uniformly exited (a) dipole array and (b) moxon array in 3D.

For the first sidelobe suppression example, we assume that there is an interference source towards the antenna array in $\varphi$ $=122^{\circ}$ direction.

Since it is known that the lower sidelobe levels than $-20 \mathrm{~dB}$ can be acceptable for most applications [15], it would be enough to suppress the sidelobe level lower than $-20 \mathrm{~dB}$ at $122^{\circ}$. But to test the power of the proposed method, we have configured the cost function (1) to limit the sidelobe level at $122^{\circ}$ with $-30 \mathrm{~dB} .70$ iterations suffice to achieve the desired pattern form.

The pattern with a suppressed sidelobe under $-30 \mathrm{~dB}$ at $122^{\circ}$ is shown in Fig. 6. The gain of the moxon antenna is $13 \mathrm{dBi}$ as shown in Fig 7. In the same figure, it is also possible to see the sidelobe suppression location indicated by the arrow in 3D.

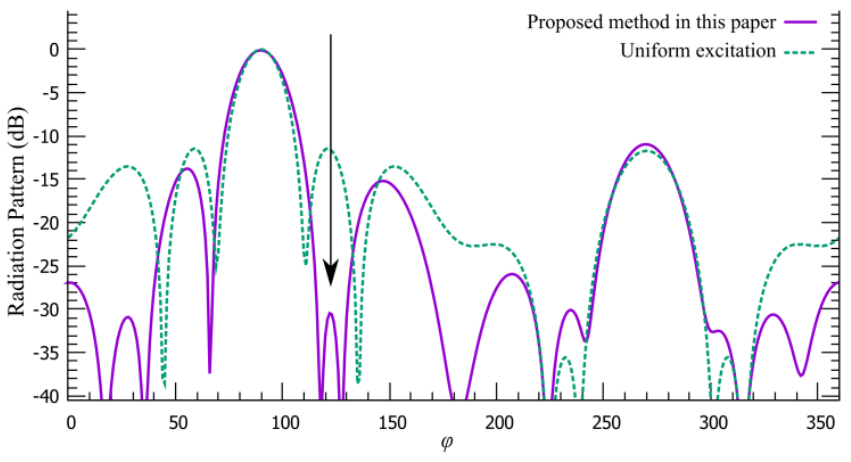

Figure 6. The moxon antenna array pattern having single sidelobe suppression at $122^{\circ}$ obtained by the proposed method and the pattern of uniformly excited moxon antenna array. 


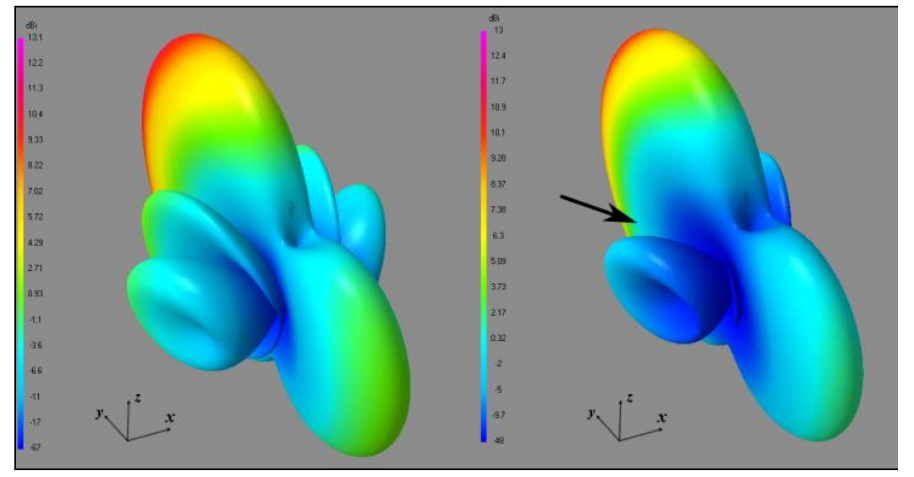

(a)

(b)

Figure 7. (a) Uniformly excited moxon antenna pattern and (b) the pattern having single sidelobe suppression at $122^{\circ}$ obtained by the proposed method.

The setup of the first example has also been used to evaluate the robustness of the algorithm. For this aim, same configuration has been executed for 30 times and the convergence values of the cost function are recorded, as well as calculated amplitude and phase values.

The patterns of moxon array computed by proposed algorithm running 30 times are presented in Fig. 8. Especially the main lobe and suppressed sidelobe are very stable for almost every run. The algorithm is able to find very similar solutions consistently. The convergence curves for these 30 run in Fig. 9 also helps to conclude that the proposed method can be considered as a robust optimization technique.

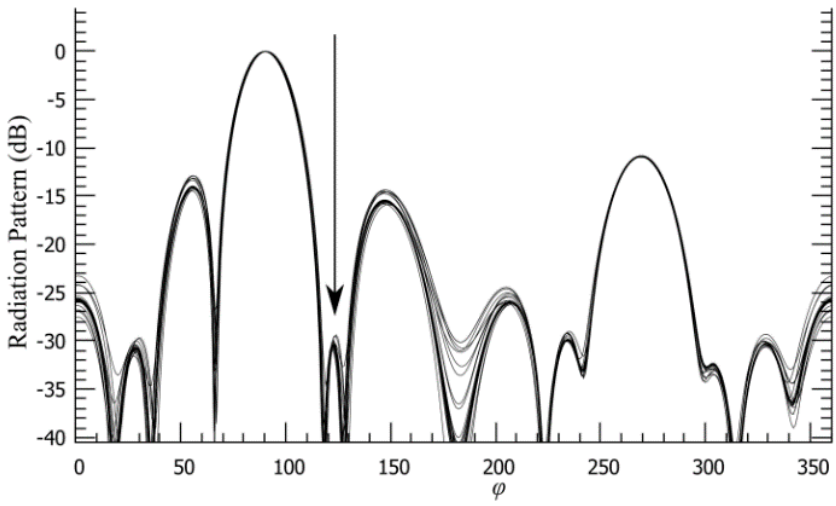

Figure 8. Patterns with a single sidelobe suppression at $122^{\circ}$ obtained by running proposed method 30 times.

For the second example, the main aim is that the sidelobe levels at $59^{\circ}$ and $152^{\circ}$ are kept under $-30 \mathrm{~dB}$ while the main lobe gain is fixed to $13 \mathrm{dBi}$,. Fig. 10 exposes that the proposed technique has successfully achieved a pattern with two suppressed sidelobe under $-30 \mathrm{~dB}$ at $59^{\circ}$ and $152^{\circ}$. Besides, Fig. 11 shows in 3D both the uniformly excited moxon array pattern and the pattern achieved by the proposed method.
It is clear from Fig. 11(a) that a pattern having $13 \mathrm{dBi}$ main lobe gain and two properly suppressed sidelobes are achieved.

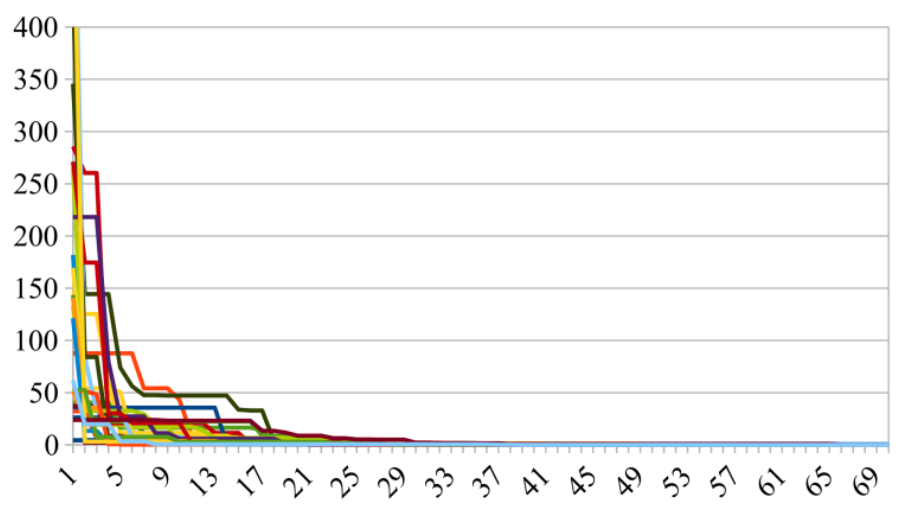

Figure 9. Convergence curves of proposed method employing DE algorithm 30 times for the first example.

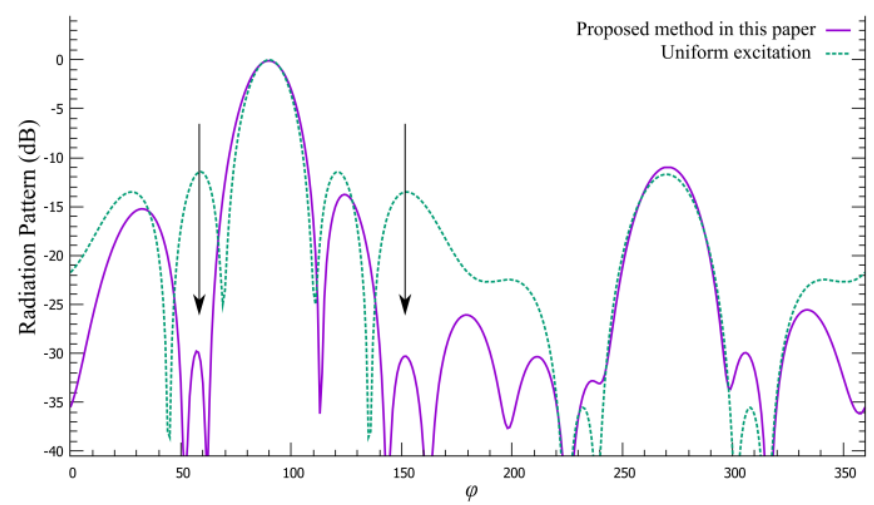

Figure 10. The moxon antenna array pattern having double sidelobe suppressions at $59^{\circ}$ and $152^{\circ}$ obtained by the proposed method and the pattern of uniformly excited moxon antenna array.

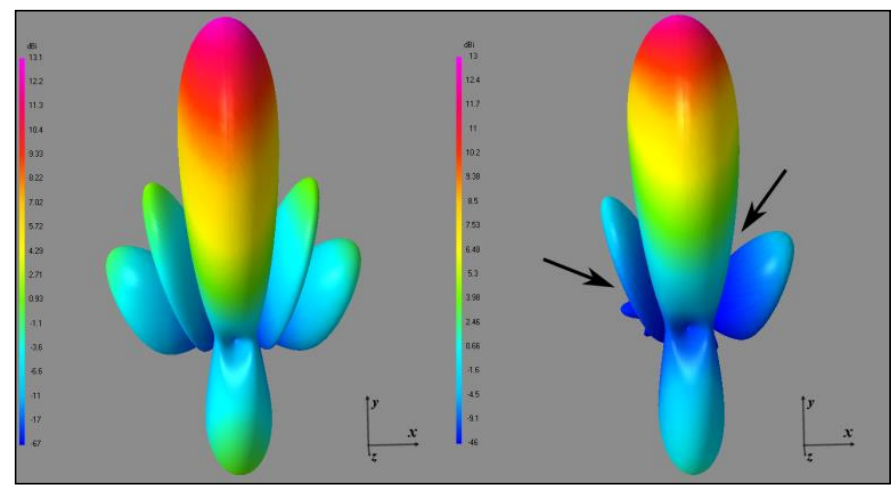

Figure 11. (a) Uniformly excited moxon antenna pattern and (b) the pattern having double sidelobe suppressions at $59^{\circ}$ and $152^{\circ}$ obtained by the proposed method.

\section{DISCUSSIONS AND CONCLUSION}

The four-element moxon antenna array introduced in this paper has a good gain and a front-to-back ratio values. It might be a better choice among the dipole alternatives for the 
applications when the high directivity is necessary in a certain direction. It also naturally owns the other advantages which individual moxon antennas have. Additionally, the moxon array has an ability to have control over the pattern shape. To do that, we have proposed a method that can exploit DE optimization algorithm and NEC2.

The proposed synthesis method has achieved the targeted results for the single and double sidelobe suppression examples. Since the cost function is built on a real simulation environment rather than simple antenna array factor, an additional procedure is not deeded to take mutual coupling effects into consideration separately. Besides, the robustness test for the first example shows that the proposed method has a very stable mechanism to find the optimal solutions.

Addition to the $2 \mathrm{D}$ plots, the examination of the $3 \mathrm{D}$ results have pointed out that the pattern attained by the proposed method is in desired form in the all planes of threedimensional space. It is natural that the technique proposed in this paper is relatively slower than the other theoretical techniques because it owns a hybrid system that includes DE and NEC2 which is a complete antenna simulation system. The results show that the method proposed in this paper, achieved more realistic results than the classical techniques [6-8]. Classical techniques use the array factor formula to optimize amplitude-only [6], phase-only [7] and amplitude and phase together [8] by assuming that the array elements are isotropic radiators. Isotropic radiators are theoretical sources rather than physical antennas. Our results are obtained by employing NEC 2 and DE techniques to control amplitude and phase values. This provides a special simulation opportunity, which can use real antenna elements. In this study, the array elements are determined as dipole antennas in our examples. Additionally, the method has been used to achieve the radiation patterns with prescribed nulls by sending optimization parameters to the original NEC2 computation engine. NEC2 was modified in [9] and [10] to design different types of antennas. However, this modification has to be done with the same language of the source codes of the NEC2 library. As a better alternative, our solutions are calculated with Python while the original code of NEC2 is C++. Our results have also confirmed that it is possible to use any programming technique to apply the method proposed in this paper. In the future works, it is planned that the proposed array will be constructed and tested with a module having four independent channels with digitally controlled frequency, phase, and amplitude. Furthermore, the antenna synthesis method introduced in this paper will be used for different antenna systems with additional parameters such as impedance bandwidth and radiation efficiency.

\section{ACKNOWLEDGMENT}

This work was supported by Research Fund of Nevsehir Haci Bektas Veli University. Project Number: BAP18F39.

\section{REFERENCES}

[1] S. Winder and J. Carr, Newnes Radio and RF Engineering Pocket Book. United Kingdom : Elsevier, 2002. [2] I. Tekin, O. Manzhura, and E. Niver, "Broadband circularly polarized antennas for UHF SATCOM," in 2011 XXXth URSI General Assembly and Scientific Symposium, 2011, pp. 1-4.

[3] N. A. Chougale, S. K. Magdum, S. D. Patel, T. D. Dongale, and P. N. Vasmbekar, "Design and development of UHF Moxon antenna," National Journal of Science and Information, pp. 93-95, 2012.

[4] J. Carson, O. Manzhura, and E. Niver, "Efficient base station antenna element for mobile communications," in Proceedings of 2014 3rd Asia-Pacific Conference on Antennas and Propagation, 2014, pp. 396-399.

[5] R. Storn and K. Price, "Differential Evolution - A Simple and Efficient Heuristic for global Optimization over Continuous Spaces," Journal of Global Optimization, vol. 11, no. 4, pp. 341-359, Dec. 1997.

[6] K. Guney and M. Onay, "Amplitude-Only Pattern Nulling of Linear Antenna Arrays with the Use of Bees Algorithm", Progress In Electromagnetics Research, vol. 70, pp. 21-36, 2007.

[7] R. L. Haupt, "Phase-only adaptive nulling with a genetic algorithm", IEEE Transactions on Antennas and Propagation, vol. 45, no. 6, pp. 1009-1015, Jun. 1997.

[8] Y. C. Chung and R. L. Haupt, "Amplitude and Phase Adaptive Nulling With a Genetic Algorithm", Journal of Electromagnetic Waves and Applications, vol. 14, no. 5, pp. 631-649, Jan. 2000.

[9] S. D. VK and S. S. Kumar, "Optimum Design of Twin Polygonal Loop Antenna Array for UHF Applications," in Advances in Computing and Communications (ICACC), 2014 Fourth International Conference on, 2014, pp. 323326.

[10] B. George and S. S. Kumar, "Optimal design of a symmetric monopole antenna with spiral geometry," in Intelligent Computational Systems (RAICS), 2013 IEEE Recent Advances in, 2013, pp. 262-267.

[11] G. J. Burke, A. J. Poggio, J. C. Logan, and J. W. Rockway, "Numerical Electromagnetic Code (NEC)," in 1979 IEEE International Symposium on Electromagnetic Compatibility, 1979, pp. 1-3.

[12] Timothy C.A. Molteno, "NEC2++: An NEC-2 compatible Numerical Electromagnetics Code", Electronics Technical Reports No. 2014-3, ISSN 1172-496X, October 2014.

[13] Moxon Rectangle Generator (MoxGen), URL: https://ac6la.com/moxgen1.html. (Visited on Nov. 19, 2018).

[14] 4nec2 Antenna Modeller and Optimizer, URL: https://www.qsl.net/4nec2/. (Visited on Nov. 19, 2018).

[15] C. A. Balanis, Antenna Theory: Analysis and Design, 4 edition. Hoboken, New Jersey: Wiley, 2016. 